\title{
Numerical Analysis of Magnetohydrodynamic Flows
}

\section{Toshio Tagawa}

Department of Aeronautics and Astronautics, Tokyo Metropolitan University, Hino, Tokyo 191-0065, Japan; tagawa-toshio@tmu.ac.jp; Tel.: +81-42-585-8662

Received: 1 February 2020; Accepted: 5 February 2020; Published: 10 February 2020

Keywords: induction equation; Hartmann number; stability analysis; alternating magnetic field

Magnetohydrodynamics (MHD) is a field of study combined by the fluid mechanics and electromagnetism. The flow of conducting materials are substantially influenced by the electromagnetic force. This mechanism has been widely applied to various industries, such as steel-making processes, semiconductor crystal growth, liquid metal blankets in nuclear fusion reactors, electromagnetic pumps, electromagnetic levitation of drop, dynamo simulation of planets, and so on. Related to these processes or phenomena, it is necessary to investigate fundamental MHD flows such as natural convection, free-surface, rotational flows, as well as the flows in ducts or pipes. Nowadays, due to the developments of both the computational resources and its techniques, more complex MHD flows are now being investigated through numerical analyses. This Special Issue focused on numerical techniques for analyzing complex MHD flows as well as their elucidation. As a matter of fact, five papers were published as the Special Issue "Numerical Analysis of Magnetohydrodynamic Flows". They can be roughly grouped into three topics: paramagnetic fluid flow under a temperature field and a strong magnetic field [1,2], MHD turbulence flow simulation including the Hall and the gyro-viscous terms [3], and numerical analysis of stability of liquid metal flow $[4,5]$.

Pleskacz and Fornalik-Wajs [1] identified the structures of low Reynolds number flow in a strong magnetic field. They focused on the magnetic field influence on forced convection in a pipe flow and analyzed the extended Graetz-Brinkman problem as three-dimensional flows. Their results exhibited the variety of possible flow structures depending on the dimensionless parameters, namely the Prandtl and the Reynolds numbers.

Song et al. [2] studied the thermomagnetic convection of paramagnetic gas in a square enclosure under no gravity field. A paramagnetic gas such as gaseous oxygen or air does not move even in the existence of temperature field under no gravity condition. However, if there is a magnetic field generated by a permanent magnet, the paramagnetic gas in an enclosure can be driven by the thermomagnetic effect. They revealed that a permanent magnet of 3.0 Tesla can enhance the Nusselt number up to 2.54 for the case of Rayleigh number $10^{5}$.

Miura [3] studied incompressible magnetohydrodynamic (MHD) turbulence under influences of the Hall and the gyro-viscous terms by means of direct numerical simulations of freely decaying, homogeneous and approximately isotropic turbulence. He revealed that the Hall and gyro-viscous terms change the energy transfer in the equations of motions to be forward-transfer-dominant while the magnetic energy transfer remains backward-transfer-dominant.

Tagawa and Song [4] studied the stability of an axisymmetric liquid metal flow driven by a multi-pole rotating magnetic field. The axisymmetric velocity profile of the basic flow for the case of an infinitely long cylinder depends on the number of pole-pairs and the Hartmann number. Depending on both the two parameters, the corresponding critical rotational Reynolds numbers for the onset of secondary flow were obtained instead of using the conventional magnetic Taylor number. The linear stability analyses revealed that the critical Reynolds number takes its minimum at a certain value of the Hartmann number. 
Tagawa [5] carried out linear stability analysis of liquid metal flow driven by a constant pressure gradient in an insulating rectangular duct under an external uniform magnetic field. A set of linearized disturbance equations for the complex amplitude was solved numerically with a finite difference method on a two-dimensional staggered mesh system. The relation among the Reynolds number, the wavenumber, the growth rate, and the angular frequency was successfully obtained for a given value of the Hartmann number as well as for a direction of external uniform magnetic field.

Finally, I would like to thank all the contributors to the Special Issue for sharing their researches, and the anonymous reviewers for donating their time to improve the quality of the submitted papers.

Conflicts of Interest: The author declares no conflicts of interests.

\section{References}

1. Pleskacz, Ł.; Fornalik-Wajs, E. Identification of the Structures for Low Reynolds Number Flow in the Strong Magnetic Field. Fluids 2019, 4, 36. [CrossRef]

2. Song, K.; Wu, S.; Tagawa, T.; Shi, W.; Zhao, S. Thermomagnetic Convection of Paramagnetic Gas in an Enclosure under No Gravity Condition. Fluids 2019, 4, 49. [CrossRef]

3. Miura, H. Extended Magnetohydrodynamic Simulations of Decaying, Homogeneous, Approximately-Isotropic and Incompressible Turbulence. Fluids 2019, 4, 46. [CrossRef]

4. Tagawa, T.; Song, K. Stability of an Axisymmetric Liquid Metal Flow Driven by a Multi-Pole Rotating Magnetic Field. Fluids 2019, 4, 77. [CrossRef]

5. Tagawa, T. Linear Stability Analysis of Liquid Metal Flow in an Insulating Rectangular Duct under External Uniform Magnetic Field. Fluids 2019, 4, 177. [CrossRef]

(C) 2020 by the author. Licensee MDPI, Basel, Switzerland. This article is an open access article distributed under the terms and conditions of the Creative Commons Attribution (CC BY) license (http://creativecommons.org/licenses/by/4.0/). 